Tôhoku Math. Journ.

28 (1976), 129-134.

\title{
HYPONORMAL OPERATORS IN VON NEUMANN ALGEBRAS
}

\author{
TAKASHI YoshINO
}

(Received May 16, 1975)

1. In [2], Foias and Kovàcs proved that a finite von Neumann algebra is characterized by the terms of contractions in it. The essential part of their proof is a trace argument. Our interest is how to prove this without using such trace argument because we can not use it in more general algebra (for example, $A W^{*}$-algebra).

We say that a bounded linear operator $T$ on a Hilbert space $H$ is hyponormal if $T^{*} T \geqq T T^{*}$, or equivalently, if $\|T x\| \geqq\left\|T^{*} x\right\|$ for all $x \in H$. If $T$ is an invertible hyponormal operator on $H$, then $T^{-1}$ is also hyponormal and $T^{*-1} T$ is a contraction (i.e., $\left\|T^{*-1} T\right\| \leqq 1$ ) in $R(T)$ which denotes the von Neumann algebra generated by $T$. On the other hand, for a contraction $T$ on $H$, we can construct a hyponormal operator in $R(T)$ associated with $T$. And there is an interesting and useful relation between hyponormal operators and contractions in a von Neumann algebra. From this point of view, it is important to study the behaviour of hyponormal operators in a von Neumann algebra and, in $\S 2$, we shall study such relation between hyponormal operators and contractions and show some applications of it.

The most effective technique being used in this section is the decomposition theorem (called canonical decomposition) of a contraction $T$ and moreover such decomposition can be done in $R(T)$. This follows from the characterization (due to [6] and also [4]) of the subspace $H^{(u)}$ on which the unitary part of $T$ acts as follows;

$$
\begin{aligned}
H^{(u)} & =\left\{x \in H:\left\|T^{k} x\right\|=\|x\|=\left\|T^{* k} x\right\|, k=1,2, \cdots\right\} \\
& =\bigcap_{k=1}^{\infty}\left\{x \in H: T^{* k} T^{k} x=x=T^{k} T^{* k} x\right\} .
\end{aligned}
$$

Concerning this decomposition, it is known that every bounded linear operator $T$ on $H$ can be written uniquely as the direct sum of a normal part and a completely non-normal part of $T$. But, in this case, known characterizations of $H^{(n)}$ on which the normal part of $T$ acts is not so precise as that of $H^{(u)}$ for the case $T$ is a contraction. In $\S 3$, we shall show that the subspace $H^{(n)}$ for the case where $T$ is hyponormal is characterized as follows; 


$$
\begin{aligned}
H^{(n)} & =\left\{x \in H:\left\|T^{k} x\right\|=\left\|T^{* k} x\right\|, k=1,2, \cdots\right\} \\
& =\bigcap_{k=1}^{\infty}\left\{x \in H: T^{* k} T^{k} x=T^{k} T^{* k} x\right\}
\end{aligned}
$$

and that this characterization does not hold for general operators. Of course, we see that this kind of decomposition of $T$ can be done in $R(T)$.

2. Hyponormal operators associated with contractions. For a von Neumann algebra $\mathfrak{2}$, the following statements are equivalent: (a) every isometry in $\mathfrak{A}$ is unitary (b) there exist sufficiently many finite normal traces of $\mathfrak{A}$.

Using this and by using a trace argument, Foias and Kovàcs [2] proved that, for any contraction $T$ in $\mathfrak{A}$, each strong limit of the sequences $\left\{T^{* k} T^{k}\right\}$ and $\left\{T^{k} T^{* k}\right\}$ is the projection in $\mathfrak{U}$ which commutes with $T$ and consequently showed the following.

THeOREM 1 ([2]). Given von Neumann algebra $\mathfrak{X}$ is finite if and only if, for every completely non-unitary contraction $T$ in $\mathfrak{A}$, the sequence $\left\{T^{k}\right\}$ converges to 0 in the strong operator topology.

However, it is of interest how to prove this without using such trace argument.

Let $T$ be a contraction on $H$. From the inequality $T^{* k}\left(I-T^{*} T\right) T^{k} \geqq 0$ it follows that the sequence $\left\{T^{* k} T^{k}\right\}$ is monotonically decreasing and hence converges strongly to a non-negative contraction. If we denote the unique non-negative square root of this contraction by $A_{T}$, then $A_{T}=0$ if and only if the sequence $\left\{T^{k}\right\}$ converges to 0 in the strong operator topology. Moreover, since $T^{*} A_{T}^{2} T=A_{T}^{2}$, we have

$$
\left\|A_{T} T x\right\|^{2}=\left\langle T^{*} A_{T}^{2} T x, x\right\rangle=\left\langle A_{T}^{2} x, x\right\rangle=\left\|A_{T} x\right\|^{2} \geqq\left\|T^{*} A_{T} x\right\|^{2}
$$

for all $x \in H$ and hence $A_{T} T$ is hyponormal. In this section, we shall consider these hyponormal operators associated with contractions. Easily we see that every isometry $V$ is a hyponormal operator $A_{V} V$ associated with $V$ itself.

Recall that a contraction $T$ on $H$ is called to be completely nonunitary if $T$ has no non-zero reducing subspace restricted to which $T$ is unitary.

LEMMA 1. For a completely non-unitary contraction $T$ on $H$, if $A_{T} T$ is normal, then $A_{T}=0$.

Proof. If $A_{T} T$ is normal, then we have $A_{T} T T^{*} A_{T}=\left(A_{T} T\right)\left(A_{T} T\right)^{*}=$ $\left(A_{T} T\right)^{*}\left(A_{T} T\right)=T^{*} A_{T}^{2} T=A_{T}^{2}$ and hence $A_{T}\left(I-T T^{*}\right) A_{T}=0$. This implies that $\left(I-T T^{*}\right)^{1 / 2} A_{T}=0$ and also $\left(I-T T^{*}\right) A_{T}=0$. Hence we have 
$\left(I-T T^{*}\right) A_{T}^{2} T=0$ and $A_{T}^{2} T-T A_{T}^{2}=0$. From this, we have $T^{* k} T^{k} A_{T}^{2}=$ $T^{* k} A_{T}^{2} T^{k}=A_{T}^{2}$ for all $k=1,2, \cdots$ and hence $A_{T}^{4}=A_{T}^{2}$. Therefore $A_{T}^{2}$ (and also $A_{r}$ ) is a projection commuting with $T$. Hence $T$ is unitary on $A_{T} H$. Since $T$ is completely non-unitary, $A_{T} H=\{0\}$ and hence $A_{T}=0$.

The following theorem follows from Theorem 1. But we shall prove this without using such trace argument as in the proof of Theorem 1.

THEOREM 2. Every hyponormal operator in a von Neumann algebra $\mathfrak{A} \subset B(H)$ is normal if and only if, for every completely non-unitary contraction $T \in \mathfrak{A}, A_{T}=0$.

PRoof. If every hyponormal operator in $\mathfrak{A}$ is normal, then, for every completely non-unitary contraction $T \in \mathfrak{N}, A_{T} T$ is also normal and hence, by Lemma $1, A_{T}=0$. Conversely, let $S$ be a hyponormal operator in $\mathfrak{A}$, then, for any complex number $\lambda, S-\lambda I$ is also a hyponormal operator in $\mathfrak{A}$. And hence we have only to prove that $S$ is normal in the case where $S$ is an invertible hyponormal operator in 2 . Let $C=$ $S^{*-1} S$, then, since the inverse of a hyponormal operator is also hyponormal, we have $\|C x\|=\left\|S^{*-1} S x\right\| \leqq\left\|S^{-1} S x\right\|=\|x\|$ for all $x \in H$, that is, $C$ is a contraction in $\mathfrak{Q}$ and $S=S^{*} C$ and hence $S=C^{* k} S C^{k}$ for all $k=$ $1,2, \cdots$. Suppose that $C=C^{(u)} \oplus C^{(0)}$ on $H=H^{(u)} \oplus H^{(0)}$ is the canonical decomposition of the contraction $C$, then $0 \oplus C^{(0)}$ is a completely nonunitary contraction in $\mathfrak{A}$ as stated in $\S 1$. Hence, by the hypothesis, the sequence $\left\{\left(0 \oplus C^{(0)}\right)^{k}\right\}$ and also the sequence $\left\{C^{(0) k}\right\}$ converges to 0 strongly. And, for any $x \in H^{(0)}$, we have

$$
\|S x\|=\left\|C^{* k} S C^{k} x\right\| \leqq\|S\|\left\|C^{k} x\right\|=\|S\|\left\|C^{(0) k} x\right\| \rightarrow 0 \text { (as } k \rightarrow \infty \text { ) }
$$

and $S x=0$. Therefore $H^{(o)} \subset N_{S}=\{x \in H: S x=0\}$. Since $S$ is invertible, $N_{S}=\{0\}$ and $H^{(0)}=\{0\}$. This implies that $C$ is unitary and $\left\|S^{*} x\right\|=$ $\left\|C^{*} S x\right\|=\|S x\|$ for all $x \in H$.

Lemma 2. Let $T$ be a contraction on $H$ and let $\left\{x_{k}\right\}$ be a sequence of unit vectors $x_{k}$ in $H$. For a real number $r>0$, if $A_{T} T x_{k}-r e^{i \theta} x_{k} \rightarrow 0$ $($ as $k \rightarrow \infty)$, then we have $T x_{k}-e^{i \theta} x_{k} \rightarrow 0($ as $k \rightarrow \infty)$.

Proof. $A_{T} T x_{k}-r e^{i \theta} x_{k} \rightarrow 0($ as $k \rightarrow \infty)$ implies that

$$
T^{*} A_{T}^{2} T x_{k}-r e^{i \theta} \cdot T^{*} A_{T} x_{k} \rightarrow 0 \text { and } A_{T}^{2} x_{k}-r e^{i \theta} T^{*} A_{T} x_{k} \rightarrow 0 \text {. } \cdots
$$

On the other hand, since $A_{T} T$ is hyponormal, $A_{T} T x_{k}-r e^{i \theta} x_{k} \rightarrow 0$ implies that

$$
T^{*} A_{T} x_{k}-r e^{-i \theta} x_{k} \rightarrow 0 .
$$

By (1) and (2), we have $A_{T}^{2} x_{k}-r^{2} x_{k} \rightarrow 0$ and also 


$$
A_{T} x_{k}-r x_{k} \rightarrow 0 \text {. }
$$

Since $r$ is non-zero, by (2) and (3), we have $T^{*} x_{k}-e^{-i \theta} x_{k} \rightarrow 0$ and, since $T$ is a contraction, we have $T x_{k}-e^{i \theta} x_{k} \rightarrow 0$ (as $k \rightarrow \infty$ ).

As an another application, we have an alternative proof of the following.

THEOREM 3 ([7]). For a completely non-unitary contraction $T$ on $H$, if $m[\sigma(T) \cap\{z \in C:|z|=1\}]=0$, then $A_{T}=0$ where $\sigma(T)$ denotes the spectrum of $T$ and $m[\cdot]$ denotes the Lebesgue measure on the unit circle.

Proof. Let $m_{2}[\cdot]$ be the two dimensional Lebesgue measure on the complex plane $C$ and let

$$
D=\left\{r e^{i \theta}: 0 \leqq r \leqq 1, e^{i \theta} \in \sigma(T)\right\} .
$$

If $m[\sigma(T) \cap\{z \in C:|z|=1\}]=0$, then, by Fubini's theorem, $m_{2}[D]=0$. Let $\sigma_{a p}\left(A_{T} T\right)$ be the approximate point spectrum of $A_{T} T$, then, by Lemma 2, $\sigma_{a p}\left(A_{T} T\right) \subset D$. Since $\partial \sigma\left(A_{T} T\right)$ (the boundary of $\left.\sigma\left(A_{T} T\right)\right) \subset \sigma_{a p}\left(A_{T} T\right)$ (see [3]) and since $D$ does not separate the plane by its construction, we have $\sigma\left(A_{T} T\right) \subset D$ and hence $m_{2}\left[\sigma\left(A_{T} T\right)\right]=0$. It is known, by [8], that, for every hyponormal operator $S$ on $H$, if $m_{2}[\sigma(S)]=0$, then $S$ is normal. Therefore $A_{T} T$ is normal. Then, by Lemma $1, A_{T}=0$.

3. Normal part of hyponormal operators. The following is a characterization of the subspace $H^{(n)}$ on which the normal part of a general operator $T$ acts, due to Apostol [1];

$$
H^{(n)}=\left\{x \in H: T^{*} T S x=T T^{*} S x \text { for all } S \in \mathscr{F}\right\}
$$

where $\mathscr{F}$ denotes the set of all finite products of $T, T^{*}$ and the identity operator I. Recently, Morrel [5] characterized the subspace $H^{(n)}$ more precisely as follows;

$$
H^{(n)}=\bigcap_{r=1}^{\infty} \bigcap_{s=1}^{\infty}\left\{x \in H: T^{* r} T^{s} x=T^{s} T^{* r} x\right\} .
$$

In the case where $T$ be hyponormal, we have the following.

THEOREM 4. The subspace $H^{(n)}$ for a hyponormal operator $T$ on a Hilbert space $H$ is characterized as follows;

$$
\begin{aligned}
H^{(n)} & =\left\{x \in H:\left\|T^{k} x\right\|=\left\|T^{* k} x\right\|, k=1,2, \cdots\right\} \\
& =\bigcap_{k=1}^{\infty}\left\{x \in H: T^{* k} T^{k} x=T^{k} T^{* k} x\right\} .
\end{aligned}
$$

Before proving the Theorem 4, we prepare several lemmas.

Lemma 3 ([9]). For a hyponormal operator $T$ on $H,\|T x\|=\left\|T^{*} x\right\|$ is equivalent to $T^{*} T x=T T^{*} x$. 
Proof. By the hyponormality, we have

$$
\|T x\|^{2}-\left\|T^{*} x\right\|^{2}=\left\langle\left[T^{*} T-T T^{*}\right] x, x\right\rangle=\left\|\left[T^{*} T-T T^{*}\right]^{1 / 2} x\right\|^{2} .
$$

For a hyponormal operator $T$ on $H$, let

$$
\mathscr{C}=\left\{x \in H:\left\|T^{k} x\right\|=\left\|T^{* k} x\right\|, k=1,2, \cdots\right\}
$$

and let

$$
\mathscr{N}=\bigcap_{k=1}^{\infty}\left\{x \in H: T^{* k} T^{k} x=T^{k} T^{* k} x\right\}
$$

then we have

Lemma 4. $\mathscr{M}$ reduces $T$ and $\mathscr{M}=\mathscr{N}$.

Proof. If $x \in \mathscr{L}$, then $\|T x\|=\left\|T^{*} x\right\|$ and, by Lemma 3,

$$
T^{*} T x=T T^{*} x \text {. }
$$

From this and by the hyponormality, $\left\|T^{2} x\right\| \geqq\left\|T^{*} T x\right\|=\left\|T T^{*} x\right\| \geqq$ $\left\|T^{* 2} x\right\|$. Since

$$
\left\|T^{2} x\right\|=\left\|T^{* 2} x\right\|,\|T(T x)\|=\left\|T^{*}(T x)\right\|=\left\|T\left(T^{*} x\right)\right\|=\left\|T^{*}\left(T^{*} x\right)\right\|
$$

and, by Lemma 3 , we have $T^{*} T(T x)=T T^{*}(T x)$ and $T^{*} T\left(T^{*} x\right)=T T^{*}\left(T^{*} x\right)$. And hence, by (1), we have

$$
T^{*} T^{2} x=T T^{*} T x=T^{2} T^{*} x \text { and } T^{* 2} T x=T^{*} T T^{*} x=T T^{* 2} x .
$$

From this and by the hyponormality, $\left\|T^{3} x\right\| \geqq\left\|T^{*} T^{2} x\right\|=\left\|T T^{*} T x\right\|=$ $\left\|T^{2} T^{*} x\right\| \geqq\left\|T^{*} T T^{*} x\right\|=\left\|T^{* 2} T x\right\|=\left\|T T^{* 2} x\right\| \geqq\left\|T^{* 3} x\right\|$. Since $\left\|T^{3} x\right\|=$ $\left\|T^{* 3} x\right\|,\left\|T\left(T^{2} x\right)\right\|=\left\|T^{*}\left(T^{2} x\right)\right\|=\left\|T\left(T^{*} T x\right)\right\|=\left\|T^{*}\left(T^{*} T x\right)\right\|=\left\|T\left(T T^{*} x\right)\right\|=$ $\left\|T^{*}\left(T T^{*} x\right)\right\|=\left\|T\left(T^{* 2} x\right)\right\|=\left\|T^{*}\left(T^{* 2} x\right)\right\|$ and, by Lemma 3 , we have $T^{*} T\left(T^{2} x\right)=T T^{*}\left(T^{2} x\right), T^{*} T\left(T^{*} T x\right)=T T^{*}\left(T^{*} T x\right), T^{*} T\left(T T^{*} x\right)=T T^{*}\left(T T^{*} x\right)$ and $T^{*} T\left(T^{* 2} x\right)=T T^{*}\left(T^{* 2} x\right)$. And hence, by (1) and (2), we have

$$
\begin{aligned}
& T^{*} T^{3} x=T T^{*} T^{2} x=T^{2} T^{*} T x=T^{3} T^{*} x, \\
& T^{* 2} T^{2} x=T^{*} T T^{*} T x=T^{*} T^{2} T^{*} x=T T^{*} T T^{*} x=T T^{* 2} T x=T^{2} T^{* 2} x \\
& \text { and } T^{* 3} T x=T^{* 2} T T^{*} x=T^{*} T T^{* 2} x=T T^{* 3} x .
\end{aligned}
$$

Repeating this, we have

$$
\begin{aligned}
& \left\|T^{k}(T x)\right\|=\left\|T^{* k}(T x)\right\|=\left\|T^{k}\left(T^{*} x\right)\right\|=\left\|T^{* k}\left(T^{*} x\right)\right\| \\
& \text { and } T^{* k} T^{k} x=T^{k} T^{* k} x \text { for all } k=1,2, \cdots .
\end{aligned}
$$

And hence $T x, T^{*} x \in \mathscr{K}$. Therefore $\mathscr{C}$ reduces $T$. Since, for any $k=$ $1,2, \cdots, T^{* k} T^{k} x=T^{k} T^{* k} x$ implies that $\left\|T^{k} x\right\|=\left\|T^{* k} x\right\|$, we have $\mathscr{N} \subset$ $\mathscr{A l}$. Conversely, if $x \in \mathscr{L}$, then, by (4), $x \in \mathscr{N}$. Therefore $\mathscr{A}=\mathscr{N}$.

Proof of Theorem 4. For each $k=1,2, \cdots,\left\{x \in H: T^{* k} T^{k} x=T^{k} T^{* k} x\right\}$ 
is a closed subspace of $H$ because it is the null space of the operator $T^{* k} T^{k}-T^{k} T^{* k}$. And hence $\mathscr{N}$ is also a closed subspace of $H$. Therefore, by Lemma 4, $\mathscr{K}$ is a closed subspace of $H$ and reduces $T$. By the construction of $\mathscr{M}$, it is clear that the restriction $T \mid \mathscr{M}$ is normal and the restriction $T \mid H \ominus \mathscr{M}$ is completely non-normal. Therefore $H^{(n)}=\mathscr{M}$.

Applying this theorem to an isometry, we have the following.

CoRollary ([6]). The subspace $H^{(n)}$ for an isometry $V$ on $H$ is characterized as follows;

$$
\begin{aligned}
H^{(n)} & =\left\{x \in H:\|x\|=\left\|V^{* k} x\right\|, k=1,2, \cdots\right\} \\
& =\bigcap_{k=1}^{\infty}\left\{x \in H: x=V^{k} V^{* k} x\right\}=H^{(u)} .
\end{aligned}
$$

The Theorem 4 does not hold for general operators.

Example. Let $T=\left(\begin{array}{ll}0 & 0 \\ 1 & 0\end{array}\right)$ on a two dimensional Hilbert space $H=$ $\{x=(\lambda, \mu): \lambda, \mu \in C\}$, then clearly $T$ is not hyponormal and is completely non-normal (i.e., $H^{(n)}=\{0\}$ ). On the other hand, we have easily that, for any $\lambda \in \boldsymbol{C}$,

$$
(\lambda, \lambda) \in\left\{x \in H:\left\|T^{k} x\right\|=\left\|T^{* k} x\right\|, k=1,2, \cdots\right\} .
$$

Therefore $H^{(n)} \neq\left\{x \in H:\left\|T^{k} x\right\|=\left\|T^{* k} x\right\|, k=1,2, \cdots\right\}$.

\section{REFERENCES}

[1] C. Apostol, Sur la partie normale d'un ensemble d'operateur de l'espace de Hilbert, Acta. Math. Academie Sci. Hung., 17 (1966), 1-4.

[2] C. FoIAS ET I. KovÂCS, Une caractèrisation nouvelle des algèbres de von Neumann finies, Acta. Sci. Math. (Szeged), 23-2, 3 (1962), 274-278.

[3] P. R. Halmos, A Hilbert space problem book, Von Norstrand Co. (1967).

[4] H. LANGER, Ein Zerspaltungssatz für operatoren im Hilbertraum, Acta. Math. Academie Sci. Hung., 12 (1961), 441-445.

[5] B. B. MORREL, A decomposition for some operators, Indiana University Math. J., 23-6 (1973), 497-511.

[6] B. Sz.-NAGY ET C. Foras, Sur les contractions de l'espace de Hilbert IV, Acta. Sci. Math. (Szeged), 21 (1960), 251-259.

[7] B. Sz.-NAGY ET C. Foias, Sur les contractions de l'espace de Hilbert V (translations bilaterales), Acta. Sci. Math. (Szeged), 23 (1962), 106-129.

[8] C. R. Putnam, An inequality for the area of hyponormal spectra, Math. Z., 116 (1970), 323-330.

[9] J. G. Stampfli, Hyponormal operators and spectral density, Trans. Amer. Math. Soc., 117-5 (1965), 469-476.

DePaRtMent OF MATHEMATICS

College of General Education

TÔHOKU UNIVERSITY

KaWaUchi, Sendai, JaPan 